\title{
The Network Computer for an Open Services Market
}

\author{
Lutz Henckel, Jiri Kuthan \\ GMD FOKUS \\ Kaiserin-Augusta-Allee 31, 10589 Berlin, Germany, \\ \{lutz.henckel,kuthan\}@fokus.gmd.de
}

\begin{abstract}
The approach of a Network Computer (NC) benefits from a client/server architecture using distributed computing capabilities but avoids the disadvantages of the required maintenance for distributed operating system and application software. In general todays available NC solutions are only applicable in an Intranet environment. The maintenance of NC operating system and applications is carried out on a central server and the NCs download the current software from the server if needed. The required management of users, storage, internet access and application usage is performed by a human administrator, who has to set up corresponding configurations manually.

Through effort centralisation for operation system and application maintenance the NC users are relieved from this task. Additionally an interconnected client/server architecture opens the possibility to access and use resources (e.g. storage, e-mail, www, ftp, internet access, etc.) over the network. These are the main reasons why enhancements of this NC approach are proposed. In doing so any provider may offer its services on an open market. The offered services can be subscribed and used by NC users acting as customers. The required enhancements encompass the definition and realisation of an Internet Services Management Protocol (ISMP) supporting dynamic negotiation of the customer/provider relationship without administrator interactions.

Besides the usage of NCs in a Small Office/Home Office (SOHO) area, it is imaginable to install corresponding equipment at public locations like airports, railroad stations, restaurants, hotels, telephone boxes, etc. Especially the mobility
\end{abstract}

The original version of this chapter was revised: The copyright line was incorrect. This has been corrected. The Erratum to this chapter is available at DOI: 10.1007/978-0-387-35388-3_42 
of NC users is supported by the enhanced approach because the users get their well-known and customized environment independent from the location where an $\mathrm{NC}$ is used.

\section{Keywords}

Network Computer, Java, Open Services Market, Internet

\section{JAVA: WRITE ONCE/RUN ANYWHERE}

The basics for a realization of Network Computers is Java [1]. Following the principal Write once/ Run anywhere the Java Development Environment (JDK) supports the realization of programs which can be executed on almost all computer or operating system platforms. Assumption for that is the availability of a Java Virtual Machine (JVM) on a target system, which is able to interpret the Byte Code generated by a Java Compiler. The JVM transforms the Byte Code into machine dependent code and then executes the program. Assuming that software has to be developed only for this virtual Java-Platform to make it executable on any system the porting effort for other platforms can be avoided. On one hand this reduces the software development costs substantially and opens smaller software manufacturers better sales opportunities. On the other hand users gain freedom during choice of a computer and operating system platform.

The second important assumption for a realization of the NC approach is the possibility to download Java-based applications over the internet for local execution. This is supported by the features to embed Java-Applets in HTML documents, which will be loaded if a user accesses the corresponding web page. Furthermore todays Web-Browsers contain a JVM as an integral component providing the direct execution of currently downloaded Java-applications. Especially these facilities open new perspectives for software distribution and maintenance.

\section{NC FOR THE INTRANET}

The client/server architecture is the most disseminated architecture for company internal computer solutions as well as the current expansion rate for the usage of internet protocols in this area indicates, that the Intranet architecture is the most promising one for the future. Todays company internal networks often consist of PCs running MS-Windows and Unix-based Servers. Besides undeniable advantages against a centralized architecture also cost disadvantages have been exposed. About $12.000 \$$ per year have to be calculated for the administration and maintenance of the PC operating system and applications for these Fat Clients. This was a result of a study about Management Strategies to Control the Rapidly Escalating Costs of Distributed Computing [3] done by the Gartner Group. 
Lowering of costs towards $2.500 \$$ per workstation can be achieved by using Thin Clients [2]. The advantage of a client/server architecture with the availability of computing capacity at any workstation remains. On the other hand the applications are administrated at a central server and are downloaded only if needed. Using Java-Technology applications may be executed on almost all platforms. As a result the effort for distribution and maintenance of applications and the operating system for every workstation is no longer necessary. Nevertheless the savings of maintenance efforts and corresponding costs have to be compared with additional needs on communication resources and costs.

In may 1996 Sun, Oracle, Netscape, IBM and Apple (SONIA) have jointly presented the Network Computer (NC) Reference Profile [4], which determines a set of Internet Standards an NC has to support. At the beginning of 1997 first NC products were introduced by IBM and Sun which can only be used in an Intranet environment. The NC equipment only supports adapters for local area networks (e.g. Ethernet, Token Ring) and does not include a hard disk or other storage media. As a consequence of this architecture a download of the operating system is required each time the $\mathrm{NC}$ is switched on.

The creation of user accounts or mailboxes as well as the assignment of OS/File servers to NCs is performed furthermore by an administrator, who has to setup corresponding configurations manually on server side. After configuration completion the $\mathrm{NC}$ users may use the following services:

- Web Browsing

- File Access and Management

- File Transfer

- Send and Receive E-Mails

- Join Usenet Discussion Groups

- Naming/Directory Services

- Internet Access

\section{NC-REQUIREMENTS FOR THE INTERNET}

For the usage of NCs in an open services market environment some concepts have to be adapted. Especially the inflexible and slow management performed by a human administrator plays an important rule. Better flexibility and automation of these management functions and other following requirements have to be taken into account for a design of a system architecture for Network Computers in an open services market:

architecture which supports load balancing to avoid performance bottlenecks.

\section{Openness}




\section{On-line Service Subscription}

The registration of NC users as well as subscription and usage of services should be effected on-line without interaction of an administrator at service provider side. Customers buying an NC don't have to decide which service provider they are going to use. The NC user has the freedom to select one of different providers offering one or multiple services and may change the provider whenever desirable.

\section{Zero Maintenance}

The NC user should not need to pay attention to the maintenance of NC operating system (NCOS) or applications. This effort should be performed automatically by the service provider.

\section{Mobility: Subscribe once/Use everywhere}

Users may use NCs at home but also in remote regions at workplace or public locations. The customized working environment (file system, applications, mailboxes, etc.) of a user should be remained without any $\mathrm{NC}$ configuration changes.

\section{Network Access}

A SOHO-NC as well as a public NC needs a network adapter to access public networks like ISDN, GSM, ADSL, etc.

\section{Performance}

Assuming the usage of Public Networks only less bandwidth is available. This has to be taken into account and may not become a knock-out criterion for the NC system architecture.

\section{Client/Server Independency}

Due to changing requirements the service provider needs the possibility to adapt its system configuration. This includes the support of a growing number of users, the provision of additional service access points in different regions, the relocation of user resources on other servers as a result of crashes or resource lacks. The adaptation of the provider-side configuration may not require configuration changes at $\mathrm{NC}$ side, which have to be performed by the $\mathrm{NC}$ user.

\section{Scalability}

The scalability of the server-side architecture has to be taken into account, so that a very large number (millions) of NC users can be supported by a service provider. The main problems in this area are the allocation of unique numerical user identifiers, the management of large databases for registered users and a distributed architecture which supports load balancing to avoid performance bottlenecks.

\section{Openness}


Prerequisite for the development of an open market for manufacturers of NC equipment as well as NC customers and service providers is the disclosure of used protocols and interfaces for services management and usage.

\section{NC SYSTEM ARCHITECTURE FOR THE INTERNET}

Considering the requirements defined above an enhanced client/server system architecture is introduced, which is shown in Fig. 1.

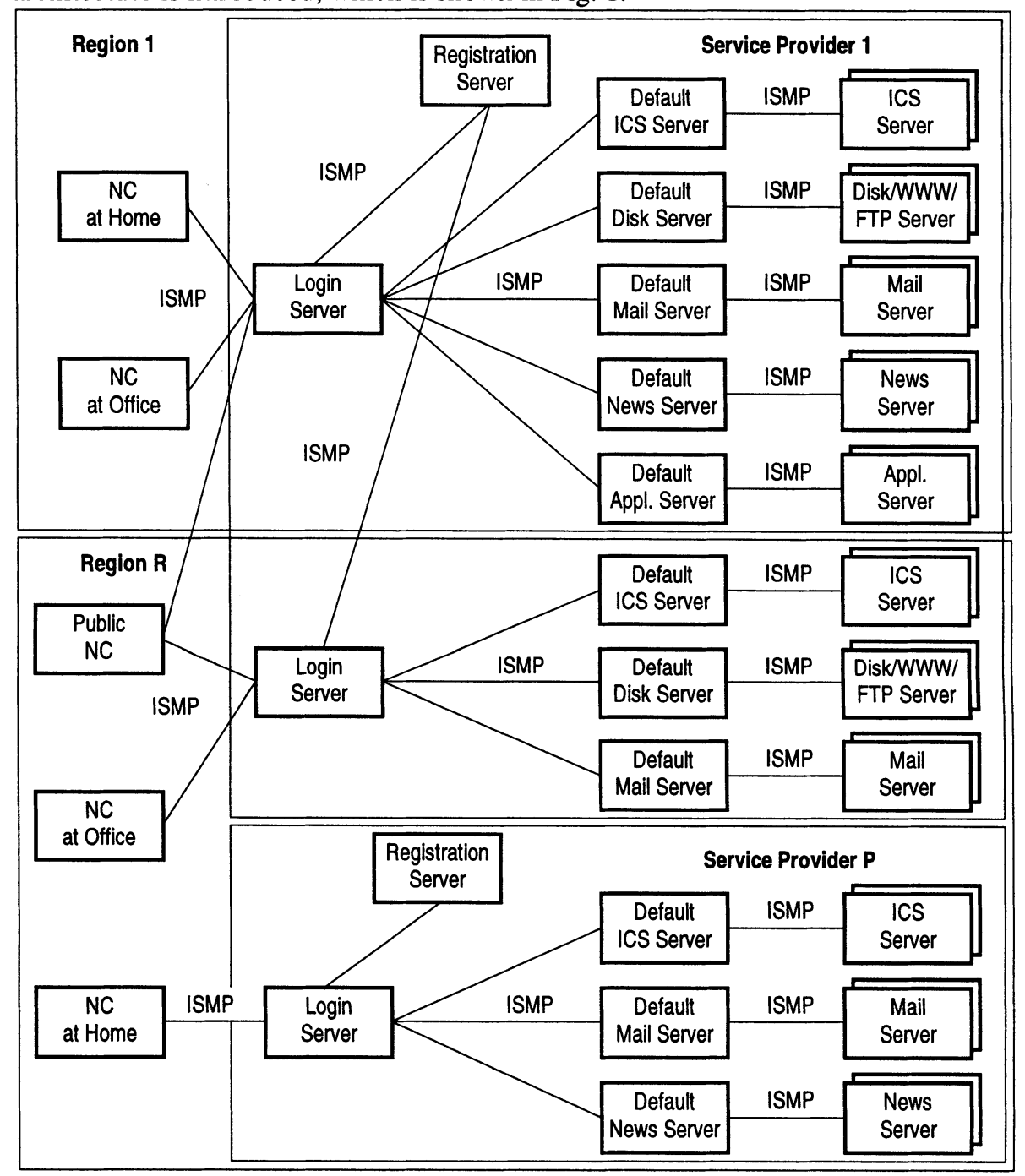

Figure 1

NC System Architecture for an Open Service Market

The enhanced services [5] provided in an open services market are the following: 
- The Internet Connectivity Service (ICS) controls Internet access and provides a persistent user environment as well as an automatic NCOS update function.

- The Disk Service provides transparent access and management of remote disk space for the storage of private data. An optional backup function is included which is performed automatically by the Disk service provider.

- The WWW Service provides transparent access and management of remote disk space on a public accessible WWW server. An optional backup function is included which is performed automatically by the WWW service provider.

- The FTP Service provides transparent access and management of remote disk space on a public accessible FTP server. An optional backup function is included which is performed automatically by the FTP service provider.

- The Mail Service enables a user to send and receive e-mails.

- The News Service enables a user to retrieve and post articles of Usenet discussion groups.

- The Application Service enables a user to download and use applications.

Before an NC user is able to use a service a registration at a provider has to be done. Therefore the user has to specify its full name, address, bank detail as well as a user name and administration password which is needed to manage the services. Furthermore for each of the above introduced services five functions are supported:

- Subscribe/Unsubscribe a service: The NC user has to specify a service password which is needed for authentication during service login and the provider allocates the requested resources.

- Login/Logout a service: The NC user authenticates itself against the provider using its service password and the provider releases the resources for access.

- Change service parameters: The NC user may change service-specific parameters, e.g. service password, amount of allocated disk space, backup interval, etc.

The client-side deploys NCs enabling users to subscribe, login and use services offered by the providers at server-side. It is assumed that several competing service providers exist and each $\mathrm{NC}$ user may subscribe several services also from different providers. Larger providers will offer their services national or international wide by providing an adequate server infrastructure in each region. Therefore load balancing can be achieved and shorter transfer routes lead to reduced response times. Finally communication costs are reduced as a result of local reachable access notes.

The actual usage of subscribed services is performed by the Internet protocols (SSAP - Service-Specific Access Protocols) which are defined in the NC Reference Profile [4]. Additionally the dynamic management negotiation between service user and provider is supported by the Internet Services Management Protocol (ISMP). 
The enhanced NC system architecture consists of the following components: The Network Computer (NC) is an economically priced system with CPU, memory, graphic, sound and network adapter as well as a keyboard, pointing device and monitor. Additionally a hard disk is included, which is used as a cache device for NCOS, HTML pages and images. The NC provides an easy-to-use graphical user interface allowing the user to register itself at different service providers as well as supporting service subscription and login. The NC Session Manager (SM) is the main application for the NC user to manage NC services. The management functions are realized by the SM using the Internet Services Management Protocol (ISMP, see below). The actual service usage is provided by other applications like a file and directory manager, web browser, ftp and e-mail clients as well as a news reader. But most of todays browsers integrate all these functions in one application, e.g. Netscape Communicator.

Using a local hard disk as a cache device a download of NCOS is not needed each time the NC is switched on. A download is only necessary if a new NCOS version is available from the service provider. As a result of this caching function the latency is reduced to a minimum which is especially important if narrowband networks are used (e.g. ISDN, GSM).

The Login Server (LS) controls the access to services of particular providers in a region. It verifies the access rights of NC users by comparing the specified user name and password with the entries of the central Provider Passwd Database which contains information about all registered users. To perform service management functions the SM of an NC communicates only with the LS, which forwards the orders to the servers (DSS - Default Service Server; SS - Service Servers) responsible for a particular service. The service provider has freedom to reconfigure its server-side infrastructure without performing any configuration effort at NC side. This is achieved by sending the address of the actual relevant service server within the reply each time a service login is initiated. This server address is then automatically used during service access initiated by SM at NC side.

The Registration Server (RS) is the central host of the service provider infrastructure where all databases reside containing information about registered NC users. The Provider Passwd Database encloses common data of NC users, e.g. full name, address, bank detail as well as user name and administration password. Additionally there exists a Service Passwd Database with service-specific data for every offered service or application containing the address of actual relevant service server and the service password. The other servers have the possibility to access these central databases (currently using NFS) to check service access rights of users or to manage user entries. The RS assigns a numerical user identification (UID) to every user, which is unique in the area the provider is responsible for. This is necessary to perform access control to file systems which are accessed and managed using NFS. An NCOS kernel-based mapping from local UID to remote ones and vice versa is necessary because a single user may get a different UID from each provider. 
The Default Service Server (DSS) accepts the subscription orders for a given service from LS and selects a Service Server (SS) having enough resources to provide the requested service. Also compliance with performance requirements is taken into account during the selection process.

The Service Server (SS) establishes the service it is responsible for and makes it available for an NC user. The SS insures that the service can only be used if it was successfully subscribed and a user specifies correct user name and password during service login phase. During service subscription an user entry is included in the Service Passwd Database. Besides service-specific parameters each entry contains the host address of the actual relevant Service Server which is delivered to NC within the reply message of ISMP during service login. This allows the provider to relocate the resources of an NC user on another Service Server. Such a relocation may be necessary if a Service Server crashes or has to be maintained. The actual service access is performed using the Service-Specific Access Protocols (SSAP) defined in [4].

After an NC user performs a registration and authentication (login) at the Internet Connectivity Service (ICS) Server the access to local servers at provider side and to other servers of the global Internet is granted.

Additionally the NC user gets access via NFS to a remote directory on ICS Server to store personal configuration data. These data contain information about services subscribed at different providers including provider name, LS host address, user name and service password as well as additional service-specific information. The management of these data is performed by the Session Manager (SM) of NC. The data deposition on a server at provider side is required to keep the actual status of subscribed services (persistent) for the user on one hand and on the other hand to have the data available independently form the location the user is working with an NC.

Each time an NC user performs a login for the Internet Connectivity Service (ICS) the ICS server sends the version number of current NCOS. The SM compares this number with the number of the current running NCOS. If the running version is elder than the version on server side the user is prompted to give its confirmation for update. On acceptance the remote file system containing the new NCOS version is mounted by the NC, automatically installed on the NCs hard disk and finally the NC is rebooted with the new NCOS. Providing this centralized maintenance of NCOS the user is relieved from this task.

The Disk/WWW/FTP Service Server creates a directory on the servers file system for the NC user during subscription phase. After verification of users access rights during login phase the directory is exported to NC which mounts it on local file system. Therefore the NC user is able to access the remote directory via Network File System (NFS) protocol just like a file system mounted from a local hard disk.

The three server types differ in their access possibilities of other Internet users. The storage on a Disk servers hard disk is only accessible via NFS for the NC user who has subscribed the Disk Service. Therefore it is only applicable for storing 
private data. In contrast to that the disk space of a WWW Service Server is simultaneously a part of the directory tree of a public accessible Web-Server. Accordingly a file system directory of an FTP Service Server is simultaneously a part of a public accessible FTP Server.

The amount of disk space which is requested by an NC user for allocation is signalled by ISMP. The Service Server maps the disk space parameters to corresponding values of the local quota system on server operating system. Therefore the quota system is used to check disk saturation and avoids violation of negotiated limits.

The Disk/WWW/FTP Service Server supports an additional backup function. During service subscription an NC user may specify a time interval which is signalled via ISMP. The service server automatically makes a backup of the directory depending on the requested interval.

The Mail Server establishes a mailbox for a user and configures an alias name, which may be specified by the user. The read access on a mailbox is supported by POP3/IMAP4 protocols and access rights are verified using the user name and password negotiated between SM and Mail Service Server during service subscription phase. Finally sending mail is supported by SMTP. The user rights for sending mail are checked using rules which are dynamically configured during login phase. For setting up these rules the negotiated user name and password are used as well as the NC host address.

The News Server controls the access to discussion groups including articles distributed by the Usenet system. The access rights of a registered user to retrieve and post articles are set if it has subscribed the News Service and performed a successful login. In the Usenet system corresponding rules have to be configured dynamically by the News Server to control these user access rights.

The Application Server controls the usage of applications which are subscribed by registered NC users. The java-based application resides in a directory of a WWW Server and may be downloaded by a browser using HTTP. The access to an application directory may only be granted for registered users which have performed a successful login. To insure this access control mechanisms of the WWW Server are used. For each application directory a database is configured with an entry for each user currently allowed to access the application. The user entries contain the user name and password negotiated via ISMP. An entry is created dynamically during login phase and is removed during logout. Each time a user accesses the application directory the WWW server asks for the user name and password. The access is granted only if a corresponding entry in the access control database was found.

Each time a user performs a login for a subscribed application the java applet is downloaded again. As a result the user can be sure that it is using the newest version available at this time. Providing this centralized maintenance of applications the user is relieved from this task.

The Internet Service Management Protocol (ISMP) is a TCP/IP based, transaction-oriented protocol for dynamic management of Internet services. It 
allows users to subscribe services and controls their usage. Especially ISMP provides reliability and authentication features to avoid incorrect service charging which may occur as a result of transaction processing over the unreliable Internet. The ISMP is used for negotiation between customer and provider (namely between SM and LS) as well as for internal communication between provider's servers (LS, RS, DSS and SS). The LS and DSS act as a dispatcher forwarding protocol data units to other server entities.

The Service-Specific Access Protocols (SSAP) are standardized Internet protocols which are determined in the NC Reference Profiles [4]. The following protocols are used to access the subscribed services after a user has performed a successful login:

- HTTP for browsing the Web and usage of java-based applications

- NFS to manage remote file systems

- SMTP to send e-mail

- POP3/IMAP4 to receive e-mail

- NNTP to join Usenet discussion groups

\section{NC PROTOTYPE REALIZATION}

A prototype implementation based on the introduced NC architecture has been developed at FOKUS. Features as on-line service subscription, Web-driven server administration, automatic $\mathrm{NC}$ administration and reliable service charging are supported. Extensive tests with the prototype have proved the concept to be a reliable means for electronic service renting and management.

Solaris-based Sun workstations powered by Sparc processors have been employed as server systems. We have taken advantage of scalability features supported by the Solaris Operating System: wide uid range, dynamic setting-up kernel properties, and robustness of the operating system guarantees operation even under high load of many on-line users. The NC server components were written in ANSI $\mathrm{C}$ and the web-driven management software was implemented by the server-side HTML-embedded scripting language PHP/FI [6].

PC with Linux has been selected for the client side because it is an inexpensive platform for which all components required in NC Reference Profile [4] (internetworking protocols, Java support, hardware requirements, etc.) are available. The Session Manager (SM) providing an easy-to-use user interface for service management, has been written in Java to achieve maximum portability (only OS-specific procedures have been implemented using shell scripts), The well-known Netscape Communicator is used to access most services managed over ISMP (application-service, e-mail, news, www, ftp). Finally a file and directory manager may be used to manage the remote file systems exported from the servers providing disk, www and ftp services. 


\section{CONCLUSION}

Current available Network Computer (NC) solutions are only applicable in an Intranet environment because the administration of users, storage, internet access and application usage is performed by a human administrator who has to set up corresponding configurations manually. This is inflexible and slow to be appropriate for an Open Services Market environment where customers may subscribe, use and change services offered by many competing service providers. Therefore the current NC approach was enhanced by a mechanism to negotiate the management parameters dynamically between customer NCs and the infrastructure of providers. This is necessary in order to automate the administration tasks for managing the relationship in an open client/server environment. The dynamic administration mechanism is realized by an Internet Services Management (ISMP) protocol, an NC Session Manager (SM) providing an easy-to-use user interface and administration servers at provider side.

Furthermore the proposed enhancements support provider-driven maintenance of the NC operating system, applications and other server-based resources. Without these maintenance tasks the usage of computers and the internet is much easier. This is the main reason why we believe to gain new customers with such an enhanced architecture, especially those people where the maintenance of Fat Clients expects too much of them or who are simply tired of wasting time with administration tasks. Additionally the concept of an open services market opens smaller companies the opportunity for outsourcing their server-based infrastructure. This avoids the needs of hiring expensive computer experts. Finally the availability of public NCs at airports, railroad stations, restaurants, hotels, telephone boxes, etc. will offer better personal mobility approximating a realization of the principal Subscribe once / Use everywhere.

\section{REFERENCES}

[1] Gosling, J., McGilton, H., The Java Language Environment, White Paper, Sun Microsystems, 1996, http://java.sun.com/nav/read/white_papers.html.

[2] Tribble, B., Java Computing in the Enterprise, What it means for the General Manager and CIO, White Paper, Sun Microsystems, 1996, http://www.sun.com/javacomputing.

[3] Gartner Group, Management Strategies to Control the Rapidly Escalating Costs of Distributed Computing, Standford, CT: Gartner Group, 1995.

[4] Apple, IBM, Oracle, Sun, Netscape, Network Computer Reference Profile, May 1996. http://www.nc.ihost.com/

[5] Henckel, L., Schilling, J., Baumgart, T., Kuthan, J., Network Computing in einem offenen DV-Dienstemarkt, Proceedings for the Workshop on Java in Telecommunications, Darmstadt (D), 12 - 13 May 1997

[6] PHP/FI, a server-side html-embedded scripting language, http://php.iquest.net/ 